\title{
The Interplay between Classical Aesthetics, Expressive Aesthetics and Persuasiveness in Behavior Modeling
}

\author{
Kiemute Oyibo \\ University of Saskatchewan \\ Saskatoon, Canada \\ kiemute.oyibo@usask.ca \\ Babatunde Olabenjo \\ University of Saskatchewan \\ Saskatoon, Canada \\ b.olabenjo@usask.ca
}

\author{
Ifeoma Adaji \\ University of Saskatchewan \\ Saskatoon, Canada \\ ifeoma.adaji@usask.ca
}

\author{
Rita Orji \\ Dalhousie University \\ Halifax, Canada \\ rita.orji@dal.ca
}

Julita Vassileva

University of Saskatchewan

Saskatoon, Canada

jiv@cs.usask.ca

\begin{abstract}
Research has shown that visual aesthetics plays a key role in the persuasiveness of behavior change support systems. However, there is limited knowledge on how much each of the two dimensions of visual aesthetics (classical and expressive) contributes to the aestheticspersuasiveness relationship. To bridge this gap, we conducted an empirical study among 669 subjects in North America to investigate: (1) the interrelationships among all three design constructs-classical aesthetics, expressive aesthetics and persuasiveness-using fitness apps featuring exercise behavior models as a case study; and (2) the moderating effect of the gender, race and exercise-type characteristics of the behavior model. Our Structure Equation Model (SEM) analysis shows that, regardless of the gender, race and exercise-type characteristics of the behavior model, both classical aesthetics and expressive aesthetics significantly impact persuasiveness. Overall, our path model explains $41 \%$ of the variance of persuasiveness, with expressive aesthetics having a stronger direct influence and mediating the influence of classical aesthetics on persuasiveness. Our findings underscore the need for designers of persuasive apps to leverage both dimensions of visual aesthetics-especially expressive aesthetics-in the design of behavior models to increase their persuasiveness as change agents.
\end{abstract}

Classical aesthetics, expressive aesthetics, persuasiveness, behavior model, fitness app, gender, race, exercise-type.

\section{INTRODUCTION}

With the advancement recorded in mobile technology, leading to the emergence of the smartphone, designers of fitness apps began equipping them with behavior models to enable users to observe and imitate the correct and effective performance of certain exercise behaviors. Behavior modeling is a behavior change technique in which "an expert shows [a] person how to correctly perform a behavior, for example, in class or on video" (p. 382) (Abraham \& Michie 2008). Behavior models in fitness apps are more or less simulated virtual coaches aimed at guiding and motivating users to engage in beneficial exercise behaviors. Traditionally, before the advent of mobile technology, leaflets had been used in health interventions to promote behavior change. Such leaflets contained a variety of information, including instructions on how to carry out a target behavior.
Factors, such as the design features of the leaflets and user characteristics, have been found to influence their effectiveness. Typical design features of these leaflets include use of pictures, framing of information, etc. (Leaviss 2010).

However, with the deep penetration of smartphones in our day-to-day lives, health promoters began leveraging their ubiquity and interactivity in promoting healthy lifestyles. In particular, the fitness domain has witnessed a dramatic increase in the number of mobile applications for promoting behavior change. Over the years, behavior modeling, in the form of instructions and visual demonstration of exercise behaviors, has remained one of the most commonly used persuasive strategies in most fitness apps on the market (Conroy et al. 2014). However, there is insufficient evidence of the 
determinants of its effectiveness in the extant literature. Moreover, in the field of persuasive technology, research has shown that a visually aesthetic app holds the potential to motivate users. This has led designers of persuasive apps in the health domain to focus on how to design visually attractive apps and artifacts to motivate behavior change. However, with respect to behavior modeling, there is a dearth of knowledge about which of the two dimensions of visual aesthetics (classical aesthetics and expressive aesthetics) has a stronger impact on the effectiveness of persuasive systems in the health domain. To bridge this gap in the body of knowledge, we conducted an empirical study $(n=669)$ to evaluate the relative impact of both dimensions of visual aesthetics on persuasiveness. We based our study on a hypothetical fitness app featuring behavior models with different design characteristics as a case study. Specifically, we aimed to answer the research questions: (1) What drives the persuasiveness of fitness apps featuring behavior models: classical or expressive aesthetics, or both? (2) Are the interrelationships moderated by the gender, race and exercise-type characteristics of the behavior model?

To answer these research questions, we based our investigation on three different variables of the behavior model design: two body-weight exercisetypes (push-up and squat), both genders (male and female) and two races (black and white). We based our study on body-weight exercise because it has become very popular among fitness enthusiasts in recent years. Specifically, in the annual global survey on trending topics in the health and fitness domain, body-weight exercise has consistently occupied one of the first two positions of the chart in the last three consecutive years (Thompson 2016; Thompson 2014; Thompson 2015). Moreover, we chose push-up and squat because they are among the most common exercises featured in most apps on the market.

The results of our SEM analysis show that both classical aesthetics and expressive aesthetics significantly impact the persuasiveness of fitness apps featuring behavior models as change agents, irrespective of the gender, race and the exercisetype characteristics of the behavior model. Overall, our path model explains about $40 \%$ of persuasiveness, with expressive aesthetics having a stronger influence on persuasiveness and mediating the influence of classical aesthetics on persuasiveness. Our findings call on designers of behavior models for motivating behavior change to leverage both dimensions of visual aesthetics, especially their expressive power of creativity, originality and novelty in creating persuasive user interface (UI) designs that can effectively motivate behavior change.
The rest of the paper is organized as follows. Section 2 focuses on background and related work. Sections 3 and 4 focus on method and result, respectively. Finally, Sections 5 and 6 dwell on the discussion and conclusion, respectively.

\section{BACKGROUND AND RELATED WORK}

In this section, we provide an overview of the concepts of aesthetics and persuasiveness.

\subsection{Aesthetics}

Aesthetics is a concept often associated with beauty (Lavie \& Tractinsky 2004). Historically, it refers to the branch of philosophy concerned with "the nature and appreciation of art, beauty and good taste" (Mastin 2008). In the eighteenth century, aesthetics took on a meaning relating to "sensory pleasure and delight" (Hekkert 2006). Over the centuries, the concept of aesthetics has continued to take on different meanings in different fields, such as art, design and architecture. In the field of human-computer interaction $(\mathrm{HCl})$, visual aesthetics refers to the visual appeal or pleasing appearance of $\mathrm{HCl}$ artifacts (Tractinsky 2002), which could be objective, subjective or both. Our study is mainly based on the subjective view of aesthetics, held by Kant (Ginsborg 2014). Lavie and Tractinsky (2004), using factor analysis, found that visual aesthetics comprised two dimensions: classical and expressive. Classical aesthetics refers to the historical notion of aesthetics, which entails orderliness, symmetry, proportion, etc. Thus, it is described by terms such as "wellorganized," "symmetrical," "clean," "clear," etc. On the other hand, expressive aesthetics reflects the creativity and originality of the designer, including his/her ability to go beyond conventions in the enrichment of his/her creation. Thus, it is described by terms such as "creative," "fascinating," "special effect," etc. In sum, classical aesthetics provides pleasure, while expressive aesthetics fosters user engagement with the UI (Lavie \& Tractinsky 2004).

\subsection{Persuasiveness}

Persuasiveness is the ability of $\mathrm{HCl}$ artifacts, e.g., persuasive apps, to influence people to change their attitude or behavior. In empirical research, perceived persuasiveness refers to a measure of "the integration of the individual's subjective evaluation of [a] system and its impact on the self' (p. 5) (Lehto et al. 2012).

\section{METHOD}

In this section, we present our research design and model, measurement instruments and participants' demographics. 


\subsection{Research Design}

In this paper, we set out to address the following two research questions:

(i) What drives the persuasiveness of fitness apps featuring behavior models: classical or expressive aesthetics, or both?

(ii) Are the interrelationships moderated by the, gender, race and exercise-type characteristics of the behavior model?

To address the above research questions, we developed a home-based fitness app, which features behavior models performing push-up and squat body-weight exercises. We chose both types of exercise because they target the upper and lower parts of the body, respectively). These models were meant to demonstrate how the target exercise behavior is performed correctly. Thus, we emphasized (highlighted) in red the respective muscle groups being worked out to make the designs more persuasive. We also provided instructions on the performance of the behavior, as shown in the top-left corner of the video in Fig. 1.

To evaluate the behavior model designs, we administered an online survey. At the beginning of the survey, we described a hypothetical fitness app for tracking physical activity to the participants. The description of the app was adapted from Meschtscherjakov et al. (2016). The app (represented by its homepage) comprises an iconbased list of twelve different types of body-weight exercises (Oyibo et al. 2016), such as push-up, squat, plank, etc. Further, we presented one of the eight versions of the behavior model designs (shown in Fig. 1) as a video to each participant in a randomized fashion. Next, we asked participants to evaluate the presented behavior model in terms of perceived classical aesthetics and expressive aesthetics. Finally, we asked participants to answer questions on the perceived persuasiveness of the fitness app.

\subsection{Measurement Instruments}

We used existing validated instruments to measure all three design constructs. To measure classical aesthetics and expressive aesthetics, we used the scale proposed by Lavie and Tractinsky (2004), as adapted by van Schaik and Ling (2009). Second, to measure persuasiveness, we used the scale proposed by Lehto et al. (2012). All three scales were assessed using a 7-point Likert scale ranging from "Strongly Disagree (1)" to "Strongly Agree (7)." Table 1 shows the scales together with their respective items. While administering the questionnaire, for the aesthetics scales, the overarching question preceding the items is: "Please rate the visual design above on the following criteria based on your first impression." Similarly, for the persuasiveness scale, the overarching question preceding the items is: "Assume the Homex App works as described at the beginning of the survey and seen in the previous video. Please answer the following general questions about yourself and regarding the app."
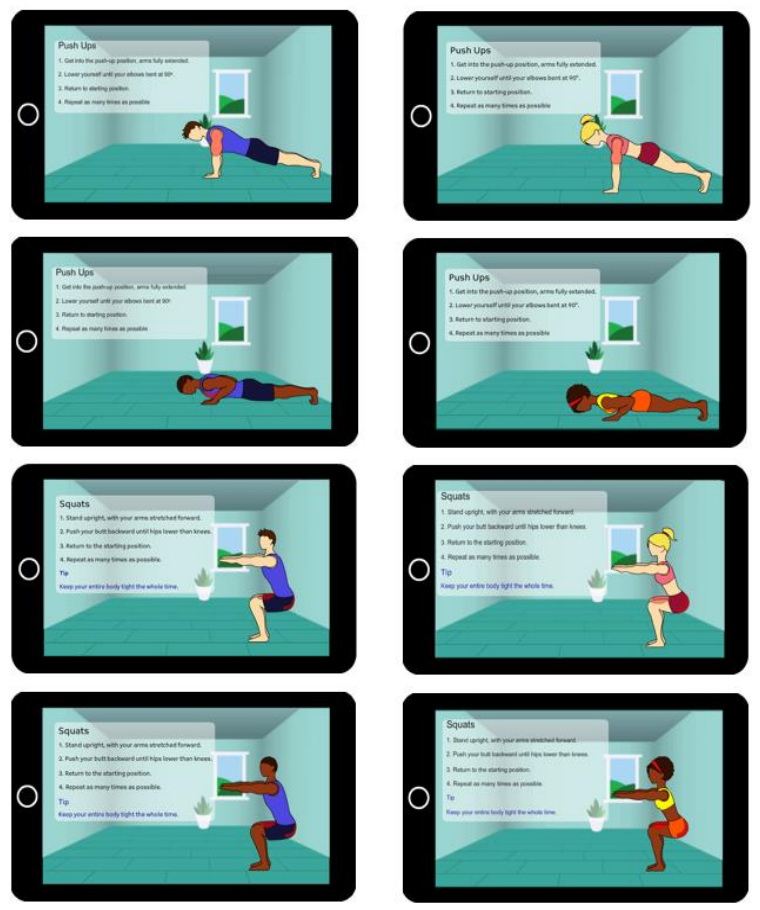

Figure 1: Race- and gender-based behavior models showing the performance of push-up and squat exercise

Table 1: Measurement instruments and their items

\begin{tabular}{l|l} 
Construct & Items in Scale \\
\hline $\begin{array}{l}\text { Classical } \\
\text { Aesthetics }\end{array}$ & 1. Visual; 2. Clean; 3. Pleasant \\
\hline $\begin{array}{l}\text { Expressive } \\
\text { Aesthetics }\end{array}$ & $\begin{array}{l}\text { 1. Fascinating; 2. Sophisticated; } \\
\text { 3. Creative }\end{array}$ \\
\hline 1. The app would influence me. \\
2. The app would be convincing. \\
Persuasiveness & $\begin{array}{l}\text { 3. The app would be personally } \\
\text { relevant for me. }\end{array}$
\end{tabular}

4. The app would make me reconsider my physical activity habits.

\subsection{Research Model}

Based on prior literature, we formulated a number of hypotheses, which are captured in the research model shown in Fig. 2. These hypotheses are stated as follows:

$\mathrm{H} 1$ : The perceived classical aesthetics of the design of the exercise behavior model will 
positively influence the perceived persuasiveness of the app.

H2: The perceived expressive aesthetics of the design of the exercise behavior model will positively influence the perceived persuasiveness of the app.

H3: The perceived classical aesthetics of the design of the exercise behavior model will positively influence its perceived expressive aesthetics.

Research (Fogg 2003; Oinas-kukkonen \& Harjumaa 2009) in persuasive design has shown that a $\mathrm{HCl}$ system that is visually attractive is more likely to be persuasive than a system that is not or less attractive. Based on this theory and given that classical and expressive aesthetics are (two) dimensions of visual aesthetics, which are related (Oyibo \& Vassileva 2017a), we hypothesize that they will both influence persuasiveness ( $\mathrm{H} 1$ and H2). Similarly, previous studies of website design (Oyibo \& Vassileva 2017b; Oyibo \& Vassileva 2016) have shown that classical aesthetics has a significant impact on expressive aesthetics. Consequently, in the fitness domain, we hypothesize that classical aesthetics will impact expressive aesthetics (H3). Finally, we adopt an exploratory approach to investigate: (1) whether the interrelationships represented in Fig. 2 are moderated by the gender, race and exercise-type characteristics of the behavior model; and (2) which of classical aesthetic and expressive aesthetics has a stronger impact on perceived persuasiveness.

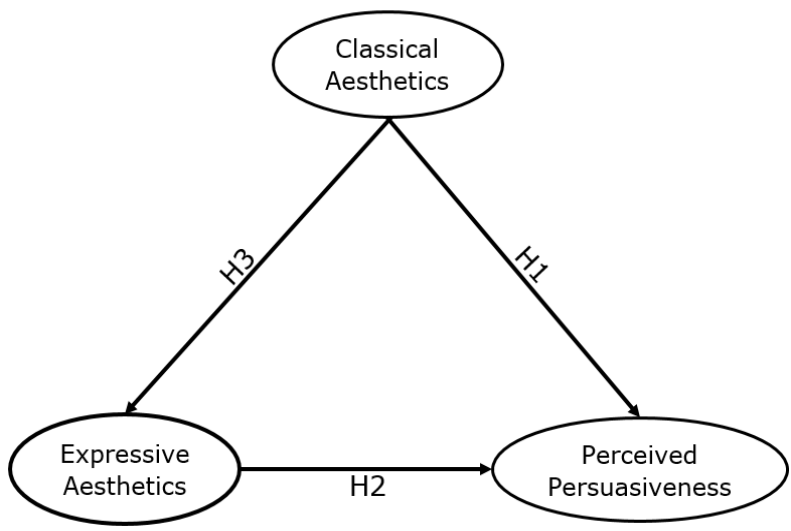

Figure 2: Hypothesized path model

\subsection{Participants}

The study was submitted to and approved by the behavioral research ethics board of the first author's university. Thereafter, we used Amazon Mechanical Turk to recruit participants resident in North America (Canada and America) to take part in an online survey. All of the participants that completed the survey were compensated with $\$ 0.6$ each in appreciation for their time. A total of 673 subjects completed the survey. However, after cleaning, we were left with 669 valid subjects for the data analysis. Table 2 shows a summary of the demographics of participants $(48.9 \%$ females and $51.1 \%$ males) and the random distribution of the behavior models among the participants.

Table 2: Demographics of participants and the distribution of behavior models among them

\begin{tabular}{|c|c|}
\hline Criterion & $($ Female, Male $)=(327,342)$ \\
\hline Age & $\begin{array}{l}18-24(56,70) ; 25-34(139,156) ; 35-34 \\
(79,77) ; 45-54(38,22) ; 54+(15,17)\end{array}$ \\
\hline Education & $\begin{array}{l}\text { Technical/Trade School }(47,39) \text {; High } \\
\text { School }(66,70) \text {; BSc }(154,162) \text {; MSc } \\
(42,54) ; \operatorname{PhD}(9,6) \text {; Others }(9,11)\end{array}$ \\
\hline Country & $\begin{array}{l}\text { Canada }(104,111) \text {; United States }(194, \\
\text { 183); Others }(26,51)\end{array}$ \\
\hline $\begin{array}{l}\text { Years on } \\
\text { the } \\
\text { Internet }\end{array}$ & $\begin{array}{l}0-3(2,2) ; 4-6(18,13) ; 7-9(20,40) \\
10+(287,287)\end{array}$ \\
\hline $\begin{array}{l}\text { Behavior } \\
\text { model } \\
\text { distribution }\end{array}$ & $\begin{array}{l}\text { BM-PU }(50,45) \text {; BF-PU }(43,39) \text {; WM- } \\
\text { PU (39, 47); WF-PU (39, 44); BM-SQ } \\
\text { (46, 42); BF-SQ }(25,46) ; \text { WM-SQ (42, } \\
\text { 41); WF-SQ }(43,38)\end{array}$ \\
\hline
\end{tabular}

$\mathrm{B}=$ Black, $\mathrm{W}=$ White, $\mathrm{F}=$ Female, $\mathrm{M}=$ Male $; \mathrm{PU}=$ Push-Up, $\mathrm{SQ}=$ Squat

\section{DATA ANALYSIS AND RESULT}

In this section, we present our data analysis, measurement models, structural models, and multigroup analysis (MGA).

\subsection{Data Analysis}

We analyzed our data using SEM. Figure 3 shows the global path model for the general population (without considering the possible moderating effect of the gender, race and exercise-type of the behavior model). The global model (as well as the submodels in Fig. 4 based on gender, race and exercise-type) were built using the consistent partial least square (PLSc) algorithm given that the path model contains (three) reflective constructs (van Riel et al. 2017). The PLSc algorithm-an updated guideline for PLS path modeling (Henseler et al. 2016)-makes the recommended correction on the results of the basic PLS algorithm to be consistent with a factor model. Specifically, the PLSc algorithm "corrects inter-construct correlations for attenuation so that the estimates of path coefficients and loadings become consistent" (p. 461) (van Riel et al. 2017).

\subsection{Measurement Model}

We evaluated the inner models and ensured the reliability and validity of the constructs in the respective path models are met (Wong 2013). The results of the evaluation are presented in the following subsections. 


\subsubsection{Indicator Reliability}

All of the reflective indicators in the respective measurement models had an outer loading greater than 0.7, except for the "clean" item which was less but greater than 0.6 .

\subsubsection{Internal Consistency Reliability}

The internal consistency reliability criterion, for the three design constructs in the path model, was assessed using the composite reliability criterion and Cronbach's alpha, both of which were greater than 0.7 .

\subsubsection{Convergent Validity}

The convergent validity criterion for the reflective constructs was assessed using the Average Variance Extracted (AVE), which was greater than 0.5 (Hair et al. 2014).

\subsubsection{Discriminant Validity}

The discriminant validity criterion was evaluated using the crossloading of each construct as well as the Heterotrait-Monotrait (HTMT) criterion. Based on the cross loading criterion, no indicator loaded higher on any other construct than the one it was meant to measure (Wong 2013). Moreover, based on the HTMT ratio-the most conservative and preferable criterion-the value for each construct was less than 0.85 and significant at $p<0.001$ (Henseler et al. 2014).

\subsection{Structural Model}

In the path models, the standardized root mean square residual (SRMR) measures the model fit. The respective SRMR values are less than the recommended threshold value of 0.08 (Henseler et al. 2016). The coefficient of determination $\left(R^{2}\right)$ measures the amount of variance of perceived persuasiveness explained by the model. For the respective models, it is approximately $40 \%$. The path coefficient $(\beta)$ is a measure of the effect of one construct on another. Overall, i.e., for the global model, both classical aesthetics and expressive aesthetics significantly influence perceived persuasiveness, with expressive aesthetics $(\beta=0.41, p<0.001)$ having a stronger direct effect than classical aesthetics $(\beta$ $=0.28, p<0.001)$ does. Moreover, classical aesthetics has a significant influence on expressive aesthetics $(\beta=0.75, p<0.001)$.

\subsection{Multigroup Analysis}

Our MGA based on the gender, race and exercisetype characteristics of the behavior model is shown in Fig. 4. As shown, the respective path coefficients in each pair of submodels replicate those of the global model. For example, in the global model, the relationship between classical aesthetics and expressive aesthetics $(\beta=0.75, p<0.001)$ is the strongest, followed by that between expressive aesthetics and perceived persuasiveness $(\beta=0.41$, $p<0.001)$. This order of the strengths of relationships is replicated in the respective pairs of submodels. For example, in the gender-based pair of submodels (Fig. 4a), the equivalent path coefficients are $(\beta=0.78 \mid 0.71, p<0.001)$ and $(\beta=$ $0.37 \mid 0.42, p<0.001$ ), respectively. These path coefficients, coupled with those of Fig. $4 \mathrm{~b}$ and Fig. $4 \mathrm{c}$, suggest that the three interrelationships generalize across the gender, race and exercisetype of the behavior model. However, in the racebased pair of submodels, the path coefficient between expressive aesthetics and perceived persuasiveness and that between classical aesthetics and perceived persuasiveness are respectively higher $(\beta=0.51, p<0.001)$ and lower $(\beta=0.17, p=n . s)$ than those of the other path models. We discuss this in Section 5. Moreover, the MGA shows there is no significant difference between the comparable path coefficients in each pair of submodels in Fig. 4.

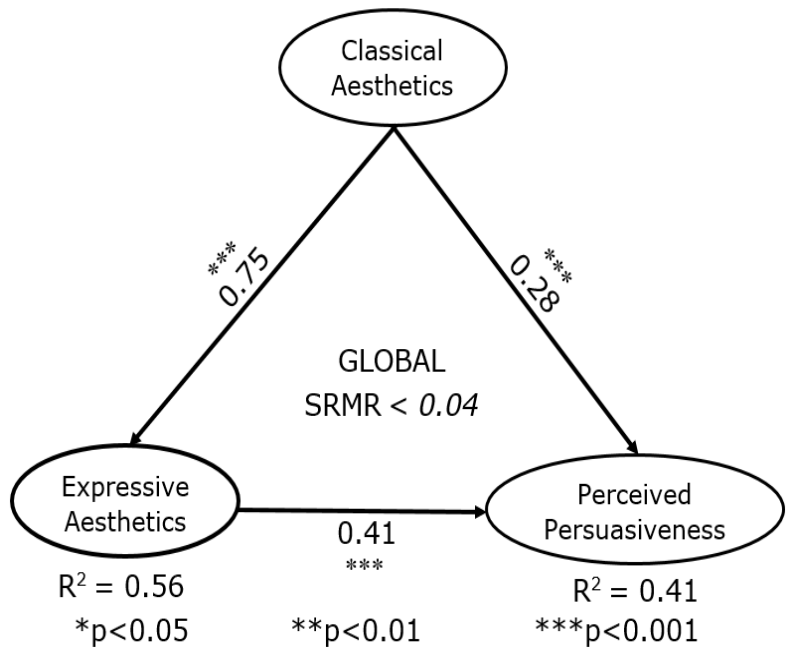

Figure 3: Global model for the evaluation of the behavior models by the general population sample

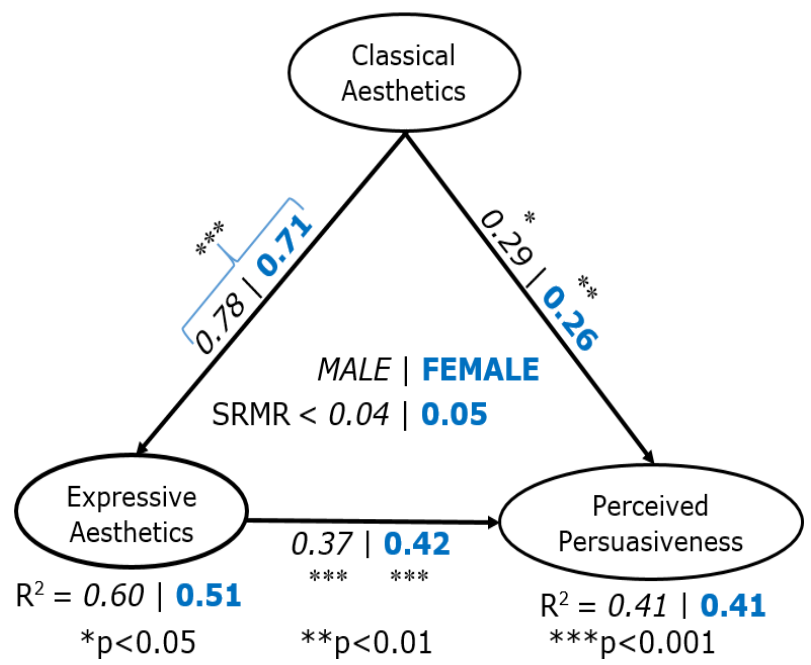

Figure 4a. Gender-based pair of submodels for male and female behavior models 


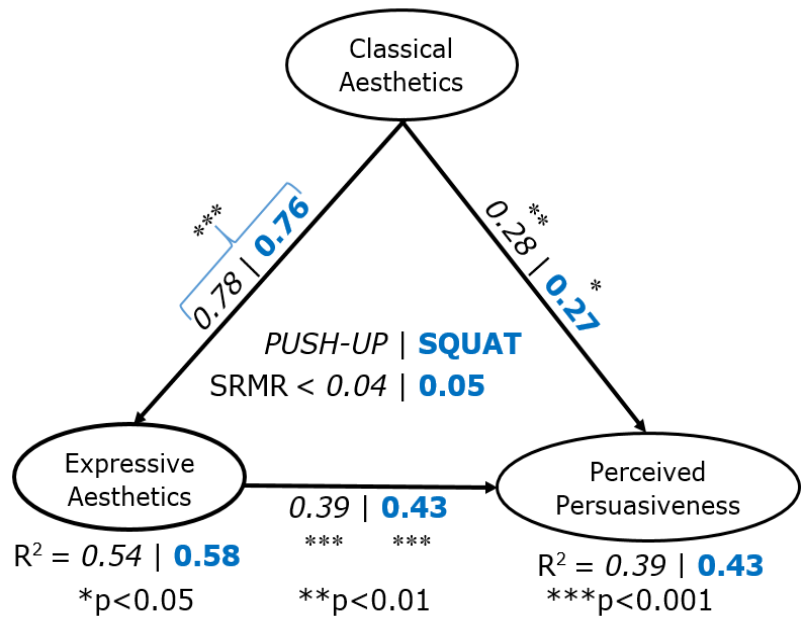

Figure 4b. Exercised-type-based pair of submodels for push-up and squat behavior models

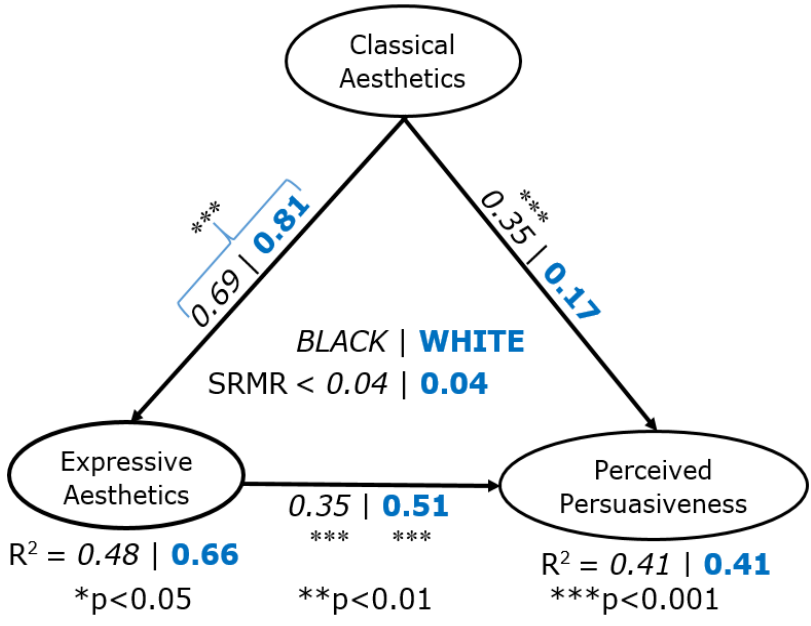

Figure 4c. Race-based pair of submodels for the black and white behavior models

\subsection{Indirect/Total Effect and Mediation Analyses}

We carried out total effect and mediation analyses on our models. The total effect analysis shows that classical aesthetics has a significant total effect on persuasiveness at the global and subgroup levels (see Table 3). Specifically, it reveals that classical aesthetics, which has a non-significant direct effect on persuasiveness ( $\beta=0.17, p=n . s)$ in the submodel for white behavior models (Fig. 4c), has a significant total effect on persuasiveness $\left(\beta_{\mathrm{T}}=\right.$ $0.58, p<0.05$ ). This leads to the conclusion that, overall, both dimensions of aesthetics significantly influence persuasiveness, irrespective of the physical and exercise-type characteristics of the behavior model. Specifically, expressive aesthetics has a stronger direct effect, while classical aesthetics has a stronger total effect as shown in Table 3. The reason classical aesthetics has a stronger total effect on persuasiveness is that it subsumes the direct effect of expressive aesthetics on persuasiveness in addition to its own direct effect on persuasiveness.
Finally, the mediation analysis shows that, in all of the path models, expressive aesthetics partially mediates the effect of classical aesthetics on persuasiveness (see Table 3). This is as a result of classical aesthetics having a significant indirect effect on persuasiveness, with the target construct's variance accounted for (VAF) by the indirect effect being greater than 20\%. Except for the submodel for black behavior models, the indirect effect accounts for at least $50 \%$ of the total effect of classical aesthetics on persuasiveness. In particular, the partial mediation by expressive aesthetics is highest for the submodel of white behavior models (VAF $=71 \%$ ). In contrast, it is least for the submodel for black behavior models $(\mathrm{VAF}=41 \%)$. We provide a possible explanation in for this difference in subsection 5.2 .

Table 3: Indirect/total effect and mediation analyses

\begin{tabular}{l|l|l|l|l} 
Effect & Direct & Indirect & Total & VAF
\end{tabular}

Global Model for the General Population Sample

\begin{tabular}{l|l|l|l|l}
$\mathrm{CA} \rightarrow$ PERS & 0.28 & 0.31 & $\mathbf{0 . 5 9}$ & 0.52 \\
\hline EA $\rightarrow$ PERS & $\mathbf{0 . 4 1}$ & - & 0.41 & - \\
\hline
\end{tabular}

Gender-based Submodels (Up: Male, Down: Female)

\begin{tabular}{l|l|l|l|l}
\hline & 0.29 & 0.29 & $\mathbf{0 . 5 8}$ & 0.50 \\
& 0.26 & 0.30 & $\mathbf{0 . 5 6}$ & 0.53 \\
\hline & $\mathbf{0 . 3 7}$ & - & 0.37 & - \\
\hline & $\mathbf{0 . 4 2}$ & - & 0.42 & - \\
\hline
\end{tabular}

Exercise-type Submodels (Up: Push-Up, Down: Squat)

\begin{tabular}{l|l|l|l|l}
\hline CA $\rightarrow$ PERS & 0.28 & 0.30 & $\mathbf{0 . 5 8}$ & 0.52 \\
\hline \multirow{2}{*}{ EA $\rightarrow$ PERS } & 0.27 & 0.33 & $\mathbf{0 . 6 0}$ & 0.55 \\
\hline & $\mathbf{0 . 3 9}$ & - & 0.39 & - \\
\hline
\end{tabular}

Race-based Submodels (Up: Black, Down: White)

\begin{tabular}{l|l|l|l|l}
\hline \multirow{2}{*}{$C A \rightarrow P E R S$} & 0.35 & 0.24 & 0.59 & 0.41 \\
& 0.17 & 0.41 & 0.58 & 0.71 \\
\hline \multirow{2}{*}{ EA $\rightarrow$ PERS } & 0.35 & - & 0.35 & - \\
\hline & 0.51 & - & 0.51 & - \\
\hline
\end{tabular}

$\mathrm{CA}=$ Classical Aesthetics, $\mathrm{EA}=$ Expressive Aesthetics, $\mathrm{PERS}=$ Persuasiveness;

$\mathrm{VAF}=$ Variance Accounted For by indirect effect: A measure of how much EA mediates the effect of CA on PERS.

$\mathrm{VAF}<0.2$ : No mediation; $0.2<\mathrm{VAF}<0.8$ : Partial Mediation; $V A F>=0.8$ : Full Mediation (Hair et al. 2014). Values in bold indicate a stronger effect between CA $\rightarrow$ PERS and $E A \rightarrow P E R S$. Values in italics are not significant at $p<0.05$ 


\section{DISCUSSION}

We presented a PLS-SEM model to investigate the influence of classical aesthetics and expressive aesthetics on the persuasiveness of fitness apps in the fitness domain. Overall, the results of our SEM analysis show that our three hypotheses (the interrelationships among the three constructs in our path model) are supported at the global and subgroup levels. This indicates that both dimensions of visual aesthetics-classical aesthetics and expressive aesthetics-influence the persuasiveness of behavior change support systems. Moreover, the total effect of both dimensions of aesthetics generalize across the gender, race and exercise-type characteristics of the behavior model. This suggests that, in the evaluation of fitness apps featuring behavior models, irrespective of the gender, race and exercise-type of the behavior model performing the target behavior, users consider both dimensions of visual aesthetics in judging how persuasive the $\mathrm{UI}$ design is. This is evident in the subjective comments (positive and negative) from a crosssection of the participants shown in Table 4.

5.1 Importance of both Classical and Expressive Aesthetics in Behavior Modeling Table 4 shows both positive and negative comments indicating participants' consideration of both dimensions of aesthetics in evaluating behavior model designs. For example, in the positive comments, we see that, in evaluating the BF-PU UI design, P97 used both dimensions of visual aesthetics. "It's very clean, and concise" refers to classical aesthetics, while "I also like that the steps are show in sequence with an animation" refers to expressive aesthetics. Specifically, P97's "clean" and "concise" descriptions of the UI capture the tradition notion of aesthetics, while the syncing of the steps (behavior instructions) with the body movement (behavior demonstration) reflects the creativity of the designer. Similarly, in evaluating the persuasiveness of the BM-PU UI design, P476 used both dimensions of visual aesthetics. "It's uncluttered and simple" reflects classical aesthetics, while "I like that it also illustrates the muscle groups being used..." reflects expressive aesthetics. Moreover, in the negative comments, we see that, in the evaluation of the BF-SQ UI design, P72 used both classical and expressive aesthetics. The first sentence of the comment, "I love how it highlights which muscles you should be activating," is a reference to expressive aesthetics. It refers to the designer's expressive power in the use of "special effect" (highlighting the specific muscle groups being worked out). Moreover, the second sentence of the comment, "The font inside the text bubble should be more centered though," refers to classical aesthetics. Specifically, it refers to the "orderliness" attribute of classical aesthetics.
Table 4: Positive and negative comments from participants on classical and expressive aesthetics.

\begin{tabular}{|c|c|}
\hline & Participants' Comments \\
\hline 1 & $\begin{array}{l}\text { "It's very clean, and concise. I also like } \\
\text { that the steps are show in sequence } \\
\text { with an animation. Often pictures are not } \\
\text { enough to fully explain the motion of an } \\
\text { exercise." [P97, WM-SQ] }\end{array}$ \\
\hline 2 & $\begin{array}{l}\text { "I like the visual design. It's uncluttered } \\
\text { and simple. It does a good job } \\
\text { demonstrating the exercise in an easy to } \\
\text { follow way and I like that it also } \\
\text { illustrates the muscle groups being } \\
\text { used..." [P476, BM-PU] }\end{array}$ \\
\hline 3 & $\begin{array}{l}\text { "The design is very simple, shows where } \\
\text { your muscles are being activated. I } \\
\text { appreciate the simple design." [P420, BF- } \\
\text { PU] }\end{array}$ \\
\hline & $\begin{array}{l}\text { "I really enjoy this visual design. It's very } \\
\text { clean and simple to follow, and I like that } \\
\text { there's both a visual instruction and text } \\
\text { instruction on how to properly do the } \\
\text { work out. I think that is a big help because }\end{array}$ \\
\hline
\end{tabular}

I've used other apps that just show someone doing the exercise, but this visual helps me fully understand how to properly form a push-up." [P455, WF-PU] "Overall the look is appealing and clean. I

5 like the moving image of the person exercising." [P480, BM-PU]

6 "Very helpful, clean informative and colorful." [P242, BM-SQ]

"I think the design is simplistic and easy

to follow, however I would not call it sophisticated or overly creative due to the very same reason..." [P40, BM-SQ]

"It looks very clean and easy to

8 understand but isn't necessarily sophisticated nor fascinating." [P207, BM-SQ]

"I love how it highlights which muscles you should be activating. The font inside the text bubble should be more centered though. The animation is great but the text makes it look a bit less design focused and sloppy." [P72, BF-SQ]

"The video is sufficient and the labeling of the actions is usable. It has a clean look

10 right now. However, the colour scheme needs a lot of work (the turquoise doesn't work for me)..." [P377, WF-PU]

$\mathrm{R}=$ Remark; $\mathrm{P}=$ Positive overall comment

$P \& N=$ Positive and negative comment

Note: Words and phrases related to the classical dimension of visual aesthetics are represented by regular bold fonts, while those related to the expressive dimension are represented by italicized bold fonts. Comments are quoted verbatim. 
Finally, the third sentence of the comment, as a way of sum up, alludes to both dimensions of aesthetics once again. "The animation is great" alludes to expressive aesthetics, while "the text makes it look a bit less design focused and sloppy" alludes to classical aesthetics.

\subsection{Relative Strength of Classical and} Expressive Effects on Persuasiveness

Based on the results of the SEM analysis, expressive aesthetics has a stronger direct effect than classical aesthetics on persuasiveness as evident in the global model and submodels. One possible explanation for this finding is that expressive aesthetics has to do with a fascinating UI design, which goes beyond simplicity, clarity and orderliness, which classical aesthetics entails (Lavie \& Tractinsky 2004). Users may have been so used to seeing or using apps with basic design features in the past. As such, they tend to take classical attributes of aesthetic for granted as compared to expressive aesthetic attributes, which only started featuring in $\mathrm{HCl}$ artifacts recently (Sonderegger et al. 2014) owing to the advancement in mobile and multimedia technologies. Perhaps, users have become less sensitive to the classical attributes of simplicity, orderliness and clarity to the point that they expect to see a more fascinating and stimulating design that goes beyond the basic app designs on the market. Moreover, they expect to see an app with more sophisticated features, including animation, variation, interactivity, etc. Thus, in their evaluation, expressive aesthetics partially mediates the influence of classical aesthetics on persuasiveness. These concerns are evident in the subjective comments from participants who expected to see a "more-than-basic" behavior model design.

For example, for PP199 (see Table 5), the design is "not creative" enough and, thus, "does not live up to the other apps available for squats and/or workouts" on the market. The same also applies to $\mathrm{P} 436$, who commented that the design is "way to[o] basic looking, many apps already look like this." No doubt, given mobile technology advancement, both participants expected to see a behavior model design that is more sophisticated than those they had previously seen. Thus, for P199, for instance, given his/her familiarity with the basic (classical) features of existing apps on the market, his/her focus in the qualitative evaluation of the behavior model design automatically shifted to expressive aesthetics (i.e. the designer's level of creativity and originality in UI enrichment). For P239 and P263, their expectations concerned exercise variations and personalization/customization, respectively. Moreover, for P514 and P324, their expectations concerned a more interactive (engaging) visual design, which is the goal of expressive aesthetics.

Table 5: Comments of participants who expected a more-than-basic behavior model design

Participants' Comments

"I feel like the visual design is not creative and it looks really basic. It looks cheaply made and like there was no effort put into the design. This design does not live up to the other apps available for squats and/or workouts." [P199, BM-SQ]

"Way to basic looking, many 2 apps already look like this." [P436, WM-PU]

\begin{tabular}{|c|c|c|}
\hline & [ ] & market. \\
\hline 3 & $\begin{array}{l}\text { "It is the easiest, but variations } \\
\text { like using a ball against the } \\
\text { wall should be shown too." } \\
{[\mathrm{P} 239, \mathrm{WM}-\mathrm{SQ}]}\end{array}$ & $\begin{array}{l}\text { Expectation } \\
\text { of exercise } \\
\text { variations. }\end{array}$ \\
\hline 4 & $\begin{array}{l}\text { "I like the design because it } \\
\text { looks very easy to follow. It } \\
\text { would be good to have more } \\
\text { diverse models though (or } \\
\text { maybe an option for the user to } \\
\text { pick their models?)." [P263, WF- } \\
\text { SQ] }\end{array}$ & $\begin{array}{l}\text { Expectation } \\
\text { of custom- } \\
\text { izable/per- } \\
\text { sonalized } \\
\text { behavior } \\
\text { model. }\end{array}$ \\
\hline 5 & $\begin{array}{l}\text { "I think the video can be more } \\
\text { graphic and interactive." } \\
\text { [P514, BM-PU] }\end{array}$ & $\begin{array}{l}\text { Expectation } \\
\text { of a more } \\
\text { interactive } \\
\text { Ul. }\end{array}$ \\
\hline 6 & $\begin{array}{l}\text { "I like the design of the girl but } i \\
\text { feel it could be more visual and } \\
\text { interactive and the empty } \\
\text { background does not do it for } \\
\text { me thats for sure." [P324, WF- } \\
\text { PU] }\end{array}$ & $\begin{array}{l}\text { Expectation } \\
\text { of a more } \\
\text { interactive } \\
\text { Ul. }\end{array}$ \\
\hline
\end{tabular}

Finally, the proposition that expressive aesthetics has a stronger direct effect on persuasiveness than classical aesthetics does due to users' prior experience and familiarity with fitness apps' basic design features plays out at a more general level in the race-based pair of submodels (Fig. 4c). Expressive aesthetics has a stronger direct effect and serves as a stronger mediator in the submodel for white behavior models than that for black behavior models (see Fig. $4 \mathrm{c}$ and Table 3). In the white submodel, the direct effect is $(\beta=0.51, p<$ $0.001)$, while, in the black submodel, it is $(\beta=0.35$, $\mathrm{p}<0.001)$. Moreover, in the former, the VAF is 0.71 (approaching full mediation), while in the latter, it is 0.41 . No doubt, most fitness apps in the marketplace (e.g., in the Apple and Android stores) feature more white than black behavior models given that they are developed in the Western world and targeted at Western users (Orji \& Moffatt 2016). As a result, due to users' relatively higher familiarity with white than black behavior models (real or virtual), they tend to emphasize expressive 
aesthetics more in the evaluation of the perceived persuasiveness of apps featuring white $(\beta=0.51, p$ $<0.001)$ than black $(\beta=0.35, p<0.001)$ behavior models. In contrast, they tend to emphasize classical aesthetics more in the latter $(\beta=0.35, p<$ $0.001)$ than in the former $(\beta=0.17, p=n . s)$.

\subsection{Summary of Main Findings}

For easy access to the results of this study, we provide a summary of the main findings as answers to our two main research questions: (1) What drives the persuasiveness of fitness apps featuring behavior models: classical or expressive aesthetics, or both? (2) Are the interrelationships moderated by the gender, race and exercise-type characteristics of the behavior model? Table 6 shows the summary of the main findings based on the total effect of the two dimensions of visual aesthetics on perceived persuasiveness. In summary, irrespective of the gender, race and exercise-type characteristics of the behavior model design, both classical and expressive aesthetics significantly influence persuasiveness $(\mathrm{H} 1$ and $\mathrm{H} 2)$. Thus, in the design of behavior change support systems, such as fitness apps featuring behavior models as change agents, both dimensions of visual aesthetics need to be given attention by designers to increase the persuasiveness of the overall app. In other words, the user interfaces of behavior change support systems should be systematically designed to be both classically aesthetic (orderly, clear and pleasant) and expressively aesthetic (creative, fascinating and stimulating) to increase their persuasiveness and the potential of being used. Specifically, designers should focus on expressive aesthetics, which has a higher potential to engage users.

Table 6: Summary of study's main findings

\begin{tabular}{l|l|l} 
No. & \multicolumn{1}{|c|}{ Hypothesis } & $\mathbf{T}$ \\
\hline $\mathrm{H} 1$ & $\begin{array}{l}\text { The perceived classical aesthetics of } \\
\text { the exercise behavior model design will } \\
\text { positively influence the perceived } \\
\text { persuasiveness of the fitness app. }\end{array}$ & $\checkmark$ \\
\hline $\mathrm{H} 2$ & $\begin{array}{l}\text { The perceived expressive aesthetics of } \\
\text { the exercise behavior model design will } \\
\text { positively influence the perceived } \\
\text { persuasiveness of the fitness app. }\end{array}$ & $\checkmark$ \\
\hline $\mathrm{H} 3$ & $\begin{array}{l}\text { The perceived classical aesthetics of } \\
\text { the design of exercise behavior model } \\
\text { will positively influence its perceived } \\
\text { expressive aesthetics. }\end{array}$ & $\checkmark$ \\
\hline $\mathrm{H} 4 *$ & $\begin{array}{l}\text { The perceived expressive aesthetics of } \\
\text { the exercise behavior model design } \\
\text { mediates the influence of perceived } \\
\text { classical aesthetics on the perceived } \\
\text { persuasiveness of the fitness app. }\end{array}$ & $\checkmark$ \\
\hline
\end{tabular}

${ }^{*} \mathrm{H} 4$ was not pre-stated but found to be true; $\mathrm{T}=$ True

\subsection{Limitations and Future Work}

There are a number of limitations in our study. The first limitation is that it is based on user perception and not actual use of the investigated app. Thus, our findings may not generalize to an actual use context, in which subjects used the app to inform their behavior change. The second limitation is that our findings are based on subjects resident in North America (Canada and United States). For this reason, our findings may not generalize to other populations or cultures. Consequently, in future work, we recommend that researchers should investigate our hypotheses in an actual application setting as well as with other populations or cultures to test the generalizability of our findings.

\section{CONCLUSION}

In this paper, we presented a path model of the perceived persuasiveness of fitness apps to determine the relative strength of influence of classical aesthetics and expressive aesthetics on persuasiveness. The path model was based on a sample of 669 subjects resident in North America (Canada and United States), who evaluated eight versions of exercise behavior model designs based on gender (male and female), race (black and white) and exercise-type (push-up and squat). The results of our SEM analysis showed that, irrespective of the gender, race and exercise-type characteristics of the behavior model design, classical aesthetics and expressive aesthetics have an overall effect on the perceived persuasiveness of fitness apps. Therefore, to make fitness apps more persuasive, designers should pay special attention to both dimensions of visual aesthetics in the design of exercise behavior models as behavior change agents. Specifically, designers should focus on the expressive dimension of aesthetics to make their apps more engaging and interactive.

\section{REFERENCES}

Abraham, C. \& Michie, S., 2008. A taxonomy of behavior change techniques used in interventions. Health psychology: official journal of the Division of Health Psychology, American Psychological Association, 27(3), pp.379-87. Available at:

http://www.ncbi.nlm.nih.gov/pubmed/1862460 3.

Conroy, D., Yang, C. \& Maher, J.P., 2014. Behavior Change Techniques in Top-Ranked Mobile Apps for Physical Activity. Am. J. Prev. Med., 46(6), pp.649-652.

Fogg, B.J., 2003. Persuasive Technology: Using Computers to Change What We Think and Do, Morgan Kaufmann.

Ginsborg, H., 2014. Kant's Aesthetics and Teleology. The Stanford Encyclopedia of 
Philosophy. Available at:

http://plato.stanford.edu/archives/fall2014/entr ies/kant-aesthetics/\%5Cninternal-

pdf://0.0.0.169/kant-aesthetics.html.

Hair, J.F. et al., 2014. A Primer on Partial Least Squares Structural Equation Modeling (PLSSEM), Sage Publications, Inc, Washington DC.

Hekkert, P., 2006. Design aesthetics: principles of pleasure in design. Psychology science, 48(2), p.157.

Henseler, J., Hubona, G. \& Ray, P.A., 2016. Using PLS path modeling in new technology research : updated guidelines. Emerald, 116(1), pp.2-20.

Henseler, J., Ringle, C.M. \& Sarstedt, M., 2014. A new criterion for assessing discriminant validity in variance-based structural equation modeling. Journal of the Academy of Marketing Science, 43(1), pp.115-135.

Lavie, T. \& Tractinsky, N., 2004. Assessing dimensions of perceived visual aesthetics of web sites. International journal of humancomputer studies, 60(3), pp.269-298.

Leaviss, J., 2010. The role of usability, usefulness and frame in persuasive health communication. Available at: http://etheses.nottingham.ac.uk/1986/.

Lehto, T., Oinas-Kukkonen, H. \& Drozd, F., 2012. Factors affecting perceived persuasiveness of a behavior change support system. Thirty Third International Conference on Information Systems, (October 2015), pp.1-15. Available at:

http://aisel.aisnet.org/icis2012/proceedings/Hu manBehavior/18/.

Mastin, L., 2008. The Basics of Philosophy. , pp.12. Available at: http://www.philosophybasics.com/branch_aes thetics.html [Accessed September 23, 2017].

Oinas-kukkonen, H. \& Harjumaa, M., 2009. Persuasive Systems Design Key Issues, Process Model, and System Features. Communications of the Association for Information Systems, 24(28), pp.485-500.

Orji, R. \& Moffatt, K., 2016. Persuasive technology for health and wellness: State-of-the-art and emerging trends. Health Informatics Journal, pp.1-26. Available at: http://www.ncbi.nlm. nih.gov/pubmed/27245673.

Oyibo, K., Ali, Y.S. \& Vassileva, J., 2016. An empirical analysis of the perception of mobile website interfaces and the influence of culture. In CEUR Workshop Proceedings. pp. 44-56.
Oyibo, K. \& Vassileva, J., 2017a. The Interplay of Aesthetics, Usability and Credibility in Mobile Website Design and the Moderation Effect of Gender. SBC Journal on Interactive Systems, 8(2), pp.4-19.

Oyibo, K. \& Vassileva, J., 2016. The Interplay of Aesthetics, Usability and Credibility in Mobile Websites and the Moderation by Culture. In Proceedings of the 15th Brazilian Symposium on Human Factors in Computer Systems IHC '16. pp. 1-10. Available at: http://dl.acm.org/citation.cfm?doid=3033701.3 033711.

Oyibo, K. \& Vassileva, J., 2017b. What Drives Perceived Usability in Mobile Web Design : Classical or Expressive Aesthetics? In 19th International Conference on HumanComputer Interaction. Vancouver, pp. 445462.

van Riel, A.C.R. et al., 2017. Estimating hierarchical constructs using consistent partial least squares. Industrial Management \& Data Systems, 117(3), pp.459-477. Available at: http://www.emeraldinsight.com/doi/10.1108/l MDS-07-2016-0286.

Sonderegger, A., Sauer, J. \& Eichenberger, J., 2014. Expressive and classical aesthetics: two distinct concepts with highly similar effect patterns in user-artefact interaction.

Behaviour \& Information Technology, 33(11), pp.1180-1191. Available at: http://dx.doi.org/10.1080/0144929X.2013.853 835.

Thompson, W.R., 2014. Worldwide survey of fitness trends for 2015: What's Driving the Market. ACSM's Health and Fitness Journal, 18(6), pp.8-17.

Thompson, W.R., 2015. Worldwide survey of fitness trends for 2016. ACSM s Health \& Fitness Journal, 19(6), pp.9-18. Available at: http://www.researchgate.net/publication/2833 11113_WORLDWIDE_SURVEY_OF_FITNES S_TRENDS_FOR_2016_10th_Anniversary_E dition.

Thompson, W.R., 2016. Worldwide survey of fitness trends for 2017. ACSM's Health and Fitness Journal, 20(6), pp.8-17.

Tractinsky, N., 2002. Visual Aesthetics. InteractionDesign, pp.1-71.

Wong, K.K., 2013. Partial Least Squares Structural Equation Modeling (PLS-SEM) Techniques Using SmartPLS. Marketing Bulletin, 24(1), pp.1-32. Available at: http://marketingbulletin.massey.ac.nz/v24/mb_v24_t1_wong. pdf. 